\title{
«La petite femme qui parlait si bien inuktitut »
}

\section{Véronique Antomarchi}

\section{OpenEdition}

Journals

Édition électronique

URL : https://journals.openedition.org/jsa/17031

DOI : 10.4000/jsa. 17031

ISSN : 1957-7842

\section{Éditeur}

Société des américanistes

\section{Édition imprimée}

Date de publication : 15 juin 2019

Pagination : 191-195

ISBN : 978-2-902715-11-4

ISSN : 0037-9174

Référence électronique

Véronique Antomarchi, « "La petite femme qui parlait si bien inuktitut » ", Journal de la Société des américanistes [En ligne], 105-1 | 2019, mis en ligne le 15 juin 2019, consulté le 04 septembre 2022. URL : http://journals.openedition.org/jsa/17031 ; DOI : https://doi.org/10.4000/jsa.17031 


\title{
« La petite femme qui parlait si bien inuktitut»
}

\author{
Véronique ANTOMARCHI *
}

Je fus l'étudiante de Michèle Therrien dans le cursus « Langue et culture inuit » à l'Inalco de 2004 à 2009, lors de cours presque particuliers. Notre rencontre a eu lieu au moment de l'inauguration de l'association Inuksuk - rue des Fêtes, au nom déjà bien annonciateur. Quatorze ans plus tard - après la soutenance de thèse de son étudiante Ariane Benoit le 22 septembre 2017 -, comme dans une ultime révérence, nous avons quitté les salons de la rue de Lille ensemble, en direction de l'ancienne salle de cours, en passant par le bureau du Centre d'études et de recherche sur les littératures et les oralités du monde (Cerlom) où Michèle était responsable de l'axe oralité. Elle paraissait alors si légère, telle un flocon de neige, déjà entre ciel et terre. Ce fut la dernière fois...

Michèle, « la petite femme qui parlait si bien inuktitut », c'est ainsi que les habitants du Nunavik, la nommaient. Elle racontait parfois comment elle avait voyagé, jeune enseignante, avec des aînés de Salluit en traîneau l'hiver et qu'ils lui parlaient, qu'elle était bercée par leur voix, par leur langue. Dès lors elle n'eut de cesse de faire connaître et aimer leur langue et leur culture à ses étudiants mais aussi au grand public. Comment lui était venue cette passion? Le Québec était alors traversé à la fois par le concept de nordicité conçu en 1961 par le géographe Louis-Edmond Hamelin et par la défense de la langue française. Peut-être que, jeune femme, elle écoutait les émissions de Glenn Gould diffusées sur Radio Canada à partir de décembre 1967. Elle a probablement entendu, alors qu'elle avait 22 ans, son émission de 59 minutes intitulée L'idée $d u$ Nord qui repose sur " l'intuition qu'un lien indissociable unit la créativité au Nord 》 (Therrien 2007a, p. 37). Elle écrit: « Glenn Gould réussit à nous donner le sentiment que nous nous trouvons dans la voiture-restaurant d'un train. » (ibid.) Elle analyse ainsi la quête du Nord chez Glenn Gould comme « une dénonciation de la vacuité de la société occidentale: il s'insurgeait contre la

\footnotetext{
* Docteure en histoire, diplômée en langue et culture inuit de l'Inalco, professeur agrégée d'histoire, université Paris Descartes, chercheuse associée au Cerlom (Inalco) et affiliée au Canthel (Paris Descartes) [veronique.antomarchi@parisdescartes.fr].
} 
massification des valeurs et de la culture, le laisser-aller de ses contemporains, leur manque de hauteur morale et de spiritualité » (ibid., p. 31). Il indiquait en effet que « quelque chose se produit chez la plupart des personnes qui vont dans le Nord. [...] Elles deviennent philosophes » (ibid., p. 36).

Michèle dresse un portrait de Glenn Gould « Homme de l'écart et de la nuit, il paraît avoir édifié une sorte de "peau" faite d'enveloppes - vestimentaires, tactiles et sonores - lui permettant d'échapper aux autres humains, comme s'il devait ignorer qu'ils étaient ses semblables » (ibid., p. 52-53). Elle ajoute: «Glenn Gould interrogeait la dimension nordique en tant que susceptible d'illuminer la part d'ombre de son moi profond, celle qui l'empêchait de se sentir en osmose avec le monde et en bonne entente avec ses semblables » (ibid., p. 51). Elle pense qu'il fait partie des « isolés » et qu'il s'interrogeait sur luimême en interrogeant les autres (ibid., p. 46). À travers ce texte, on comprend que cette quête du Nord rejoint « l'idéal d'un univers saturé de messages chargés de sens. [...] Le Nord ordonne la pensée et suscite des sentiments d'élévation morale. Le temps et la contingence étant abolis, reste la contemplation de la beauté et de l'harmonie absolues » (ibid., p. 31).

Michèle était probablement habitée elle aussi par une quête du Nord, à tel point qu'elle partit là-bas, une licence de philo en poche, à l'âge de 24 ans. Elle revient dans l'introduction de son premier ouvrage ${ }^{1}$ sur son parcours et l'origine de sa recherche: "Le choix d'un thème de recherche laisse transparaître une série de motivations affectives et intellectuelles, raconte dans les limites qui sont les siennes, une histoire. » (Therrien 1987, p. 1). Selon sa sœur, il ne pouvait pas en être autrement, le Nord était en elle depuis toujours ${ }^{2}$. Sa volonté d'apprendre la langue inuit est liée à son expérience de jeune enseignante à Salluit (NunavikNord Québec) en 1969. Elle vécut alors une prise de conscience brutale dans ce territoire situé à 2000 kilomètres de Montréal où elle assista à la reproduction des querelles linguistiques qui opposaient Québec et Ottawa avec un affrontement entre langues dominantes et langues dominées. Elle voulut apprendre la langue car elle ressentait un malaise entre l'interprète, les parents, les élèves. Elle explique: «J'ai alors abordé la langue inuit sans approche livresque, animée par l'impérieux besoin de me sentir moins à l'écart de la société dans laquelle je vivais. » (ibid.) Ce sentiment de solitude, de mise à l'écart semble être un sentiment qui a traversé Glenn Gould mais sans doute aussi Michèle. Elle a consacré sa vie à cette langue et aux Inuit: « J'avoue que la langue des Inuit depuis mes tout premiers contacts avec elle, exerce sur moi un attrait aussi puissant que durable », écrit-elle à l'âge de 42 ans (ibid.). La parole, et en particulier l'oralité furent au cœur de ses préoccupations. Elle indiquait souvent que la parole devait circuler. En tant qu'enseignante, la puissance de la parole

1. Le corps inuit, publié en 1987, version raccourcie de sa thèse.

2. Communication Gisèle Therrien, 25 mai 2018. 
était au centre de son activité professionnelle. En tant que chercheuse, ce fut le thème central de son travail. Elle aimait ponctuer ses textes de références à la poésie autochtone citant par exemple le poème d'Henriette Lynge Kristiansen du Groenland:

Les paroles te sont nécessaires

Elles te disent qui tu es et où tu es.

Nous parcourons une terre gelée

Recouverte l'hiver par l'obscurité

Les paroles sont des guides qui traversent la nuit. (Therrien 2007b, p. 19)

Louis-Jacques Dorais lui rendait hommage dans La parole inuit publiée en 1996: «Rares sont les auteurs qui [...] comme Michèle Therrien de Paris s'adonnent à des analyses ethnolinguistiques en profondeur » (Dorais 1996, p. 3).

Elle travailla avec une des personnalités essentielles du Nunavik Taamusi Qumaq dont elle restitue les entretiens dans un article consacré à la maladie. Elle revient sur sa méthode de travail: « Avant de m'entretenir avec Taamusi Qumaq, mon interlocuteur principal, j’ai réfléchi à ce qui serait susceptible d'aviver son savoir. Je me suis plongée dans son dictionnaire pour y lire les définitions relatives à la santé et à la maladie, prendre le pouls de sa pensée, travailler dans le discontinu (les mots du dictionnaire) [...] avant d'être confrontée à la réalité de la parole échangée. J'ai également lu, avec une attention particulière au lexique utilisé, ce que d'autres Inuit avaient écrit, dans divers ouvrages ou revues. J'ai proposé à mon interlocuteur de procéder selon une approche ethnolinguistique en recherchant les corrélations entre les concepts et leur expression linguistique, approche qui se situe à bien des égards, à proximité de celles des archéologues. Il est en effet possible de considérer les syntagmes inuit comme des témoins façonnés que l'on fouille, manipule pour en extraire les éléments constitutifs, et que l'on soumet à l'analyse comparative pour en reconstituer le sens global ${ }^{3}$. »

Michèle rappelle que les Inuit s'entendent sur un ensemble de valeurs protégeant l'individu de la maladie. Il s'agit d'avoir une attitude de confiance à l'égard de la vie car « celui qui sait chanter et rire ne ressasse pas ses ennuis ${ }^{4}$ ». Le souci du bien-être de l'autre est également essentiel comme le lui confirmait Taamusi Qumaq: «Toute bonne vie comporte des piniarniq. Tu es venue m'interroger et je suis rémunéré pour le travail que je fais, c'est piniarniq. [...] Piniarniq désigne quelque chose de bon et d'utile que nous faisons pour quelqu'un ou pour notre corps. » (Therrien 1995, p. 82).

En tant qu'historienne, j'aimerais évoquer plus particulièrement la contribution de Michèle Therrien à la protection d'un patrimoine photographique en danger.

3. Entretiens avec Taamusi Qumaq (Therrien 1995, p. 71).

4. Rasmussen, cité par Therrien (1995, p. 82). 
Elle avait consulté dans les archives privées de la société Revillon Frères la collection de photographies de Robert Flaherty que le cinéaste avait prises dans les années vingt pendant le tournage de Nanouk of the North, considéré en 1922 comme le premier documentaire de l'histoire du cinéma. Ces clichés sont publiés en partie à la fin de son ouvrage Le corps inuit en 1987. Puis, en 2000, elle revient sur l'histoire de la société Revillon en traduisant de l'anglais l'ouvrage de Thierry Mallet Kakoot. Récits du pays des caribous (2000).

Ainsi, elle a permis de sauvegarder près de 2000 clichés des archives privées de la société de fourrures Revillon. Certains sont réalisés par Robert Flaherty et Thierry Mallet mais la plupart d'entre eux émanent des employés de la société. Ce corpus forme une source documentaire exceptionnelle à la fois sur la vie quotidienne des postes de traite, sur les autochtones, sur le regard occidental porté sur ces territoires et leurs habitants dans les années 1920-1936. En déposant les copies à l'Institut culturel Avataq à Montréal et en réalisant un inventaire à la demande des Archives nationales du Canada, Michèle Therrien a protégé ce patrimoine photographique. Les trois quarts des clichés, explique Michèle, avaient été classés soigneusement dans des albums « à l'aspect intimiste, révélant la conscience des membres de la famille Revillon et par extension celle du personnel de participer à une aventure susceptible de laisser une trace dans l'histoire » (Mallet 2000, p. 141). Michèle racontait que par la suite la vente de la société Revillon aurait entraîné la perte de la trace des originaux de ce trésor photographique, moisissant peut-être au fond d'un garage. C'est le sort de bien des photographies appartenant à des fonds privés lorsqu'elles ne sont pas léguées à des Archives nationales.

En 2008, Michèle dirigea aux éditions Karthala un ouvrage intitulé Paroles interdites. La parole interdite rejoint le non-dit de la maladie, Michèle ne voulait pas que l'on communique sur la maladie qui la touchait: cela devait rester un secret partagé par un petit nombre de personnes à qui elle avait demandé de garder le silence. Elle avait du mal à parler de sa maladie. Avec le recul, ce titre paraît prémonitoire, la vie se montre parfois ironique... En langue inuit, Nord se dit "taqraq »-1'ombre -, un terme voisin de "tarniq » - la composante immortelle de l'être humain que seuls les chamanes peuvent entrevoir. Comme Michèle l'écrivait dans Le corps inuit: « la cessation de la vie n'est jamais un total anéantissement mais la négation d'un certain espace et d'un certain temps. [...] L'individu, privé de certaines facultés, habite ailleurs comme à la suite d'un déménagement, et les vivants peuvent parfois l'entendre sans le voir. On dit qu'il a cessé d'être un humain vivant inuugunnaituq ou qu'il a cessé d'être parfaitement visible, nittagunnaituq comme lorsque les traits du paysage s'estompent en période de brouillard » (Therrien 1987, p. 158-159).

Dans un coin de mon jardin, tout au long de cette année, un érable a pris racine de façon spontanée. J'y vois comme une présence. 
« La petite femme qui parlait si bien inuktitut »

\section{Références citées}

Dorais Louis-Jacques

1996 La parole inuit. Langue, culture et société dans l'Arctique nord-américain, Peeters (Arctique, 3 ; Selaf, 354), Paris.

Mallet Thierry

2000 Kakoot. Récits du pays des caribous, traduit de l'anglais, présenté et annoté par Michèle Therrien, commentaire ethnohistorique de Yvon Csonka, Septentrion, Québec/Sillery.

THERRIEn Michèle

1987 Le corps inuit. Québec arctique, SELAF/Presses universitaires de Bordeaux (Arctique, 1), Paris.

1995 «Corps sain, corps malade chez les Inuit, une tension entre l'intérieur et l'extérieur », Recherches amérindiennes au Québec, 25 (1), p. 71-84.

2007a «Glenn Gould et le Nord», in Ghyslaine Guertin (dir.), Variations sur des thèmes de Gould, Momentum, Montréal, p. 25-56.

2007 b «n art contemporain », Vacarme, 39, p. 18-19. 
